\title{
Autophagy is activated to protect renal tubular epithelial cells against iodinated contrast media-induced cytotoxicity
}

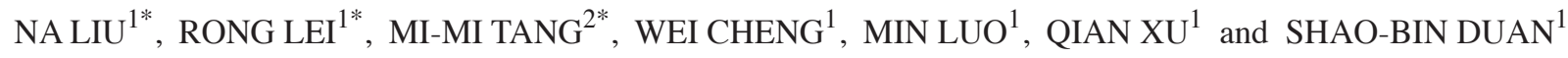 \\ ${ }^{1}$ Department of Nephrology, Second Xiangya Hospital of Central South University, Nephrology Institute of \\ Central South University, Center of Kidney Disease and Dialysis In Hunan Province, Changsha, Hunan 410011; \\ ${ }^{2}$ Department of Pharmacy, Xiangya Hospital, Central South University, Changsha, Hunan 410008, P.R. China
}

Received September 19, 2016; Accepted July 14, 2017

DOI: $10.3892 / \mathrm{mmr} .2017 .7599$

\begin{abstract}
With the steady increase in the use of various contrast-related technologies in recent years, contrast-induced nephropathy has received significant attention from clinicians and researchers. The present study aimed to determine the potential role of autophagy in iodinated contrast media (CM)-induced cell injury and apoptosis in human proximal renal tubular epithelial HK-2 cells. The present study used the iodinated CM iohexol (200 mg iodine $/ \mathrm{ml}$ ) to treat HK-2 cells for $6 \mathrm{~h}$, in order to establish a damaged cell model. The autophagy inhibitor 3-methyladenine (3-MA; $10 \mathrm{mM}$ ) was used to inhibit the formation of autophagosomes. Immunofluorescence assay and transmission electron microscopy (TEM) were used to observe the formation of autophagosomes in the cytoplasm. The protein expression levels of microtubule-associated protein 1A/1B-light chain 3 (LC3)-II and autophagy-related protein 7 (Atg7) were detected by western blotting. The cytotoxicity of CM was evaluated by MTT assay and lactate dehydrogenase release, and cell apoptosis was detected by flow cytometry. Increased formation of autophagosomes and autophagic vesicles was observed under TEM in HK-2 cells following iohexol treatment for $6 \mathrm{~h}$. Immunofluorescence assay demonstrated that iohexol increased LC3-II expression in HK-2 cells. Furthermore, the expression levels of LC3-II and Atg7 in HK-2 cells were significantly upregulated by iohexol administration; however, LC3-II and Atg7 expression was decreased by 3-MA treatment. These results provided evidence to suggest that autophagy is activated in response
\end{abstract}

Correspondence to: Professor Shao-Bin Duan, Department of Nephrology, Second Xiangya Hospital of Central South University, Nephrology Institute of Central South University, Center of Kidney Disease and Dialysis In Hunan Province, 139 Renmin Road, Changsha, Hunan 410011, P.R. China

E-mail: duansb528@qq.com

\section{${ }^{*}$ Contributed equally}

Key words: acute kidney injury, contrast media, autophagy, apoptosis, renal tubular epithelial cells to iohexol-induced cell injury and apoptosis, and may exert a renoprotective role in $\mathrm{HK}-2$ cells.

\section{Introduction}

Contrast-induced nephropathy (CIN) is a severe complication that occurs in response to intravascular administration of radio contrast media (CM), and is the third most common cause of hospital-acquired acute kidney injury (AKI) (1). CIN not only prolongs hospitalization and increases the medical burden, but also significantly increases the risk of cardiovascular events, long-term chronic kidney disease and mortality (2). A previous study indicated that iodinated CM-induced cell apoptosis serves an important role in the pathogenesis of CIN (3). In addition, CM induces ischemia, hypoxia, oxidative stress and toxicity, resulting in $\mathrm{HK}-2$ cell apoptosis and kidney damage (4). Our previous study demonstrated that iohexol is able to cause injury and apoptosis of HK-2 cells in a time- and dose-dependent manner (5). Despite numerous efforts being made to reduce the risk of $\mathrm{CIN}$, effective treatment strategies are required and detailed mechanisms regarding the pathophysiology of CIN remain to be elucidated.

Autophagy is a cellular process that results in the removal of damaged organelles and the recycling of cellular proteins (6). In the majority of cases, autophagy serves an important role in maintaining cell survival and tissue homeostasis $(7,8)$. During autophagy, the cytosolic form of microtubule-associated protein 1A/1B-light chain 3 (LC3), LC3-I, is converted to LC3-phosphatidylethanolamine conjugate (LC3-II) by the E1-like enzyme autophagy-related protein 7 (Atg7), which is recruited to autophagosomal membranes (9).

A previous study demonstrated that there was a close association between autophagy and apoptosis; autophagy reduces cell apoptosis and serves a protective role against AKI, including ischemia/reperfusion- or cisplatin-induced kidney injury (10). However, the role of autophagy in iohexol-induced HK-2 cell apoptosis remains unclear. Therefore, the present study was conducted to investigate the role of autophagy in iohexol-induced HK-2 cell injury and apoptosis, with the aim of providing a potential therapeutic strategy for CIN. 


\section{Materials and methods}

Cell culture. HK-2 cells were obtained from the Kidney Disease Laboratory of Second Xiangya Hospital of Central South University (Changsha, China). The cells were cultured at $37^{\circ} \mathrm{C}$ in a humidified atmosphere containing $5 \% \mathrm{CO}_{2}$ in Dulbecco's modified Eagle's medium (DMEM)/F12 (Gibco; Thermo Fisher Scientific, Inc., Waltham, MA, USA) supplemented with $10 \%$ fetal bovine serum (FBS; Gibco; Thermo Fisher Scientific, Inc.) and antibiotics (100 IU/ml penicillin and $100 \mu \mathrm{g} / \mathrm{ml}$ streptomycin). Iohexol was purchased from Nycomed China (Shanghai, China) and 3-methyladenine (3-MA) was purchased from Sigma-Aldrich; Merck KGaA (Darmstadt, Germany).

Cell treatment. HK-2 cells were incubated in FBS-free DMEM/F12 for $12 \mathrm{~h}$. The establishment of a damaged cells model was performed as previously reported (11). 3-MA $(10 \mathrm{mM})$ was used to inhibit the formation of autophagosomes. The cells were divided into the following four groups, all of which were cultured at $37^{\circ} \mathrm{C}$ : Control group, in which cells were cultured in DMEM/F12 supplemented with $1 \%$ FBS; CM group, in which cells were treated with $200 \mathrm{mg}$ iodine $/ \mathrm{ml}$ iohexol for $6 \mathrm{~h}$; 3-MA group, in which cells were treated with $10 \mathrm{mM}$ 3-MA for $30 \mathrm{~min}$; and CM + 3-MA group, in which cells were pretreated with $10 \mathrm{mM} 3-\mathrm{MA}$ for $30 \mathrm{~min}$ prior to treatment with $200 \mathrm{mg}$ iodine $/ \mathrm{ml}$ iohexol for $6 \mathrm{~h}$.

Transmission electron microscopy (TEM). HK-2 cells were cultured in 6-well plates at a density of $1-5 \times 10^{5} / \mathrm{ml}$, and were treated as aforementioned. The cells were digested and collected routinely. Subsequently, the cells were suspended and fixed with $2.5 \%$ glutaraldehyde in $0.1 \mathrm{mM}$ PBS $(\mathrm{pH} 7.4)$ at $4^{\circ} \mathrm{C}$ for $2 \mathrm{~h}$. After washing twice with PBS, cells underwent conventional dehydration, osmosis, embedding, sectioning and staining, as previously described (12). Cell ultrastructure was observed under a Philips 300 electron microscope (Philips Healthcare, Amsterdam, The Netherlands). TEM was used to detect the effects of iohexol on HK-2 cell autophagy.

Immunofluorescence assay. Cells were cultured on polylysine-coated glass slides (Sigma-Aldrich; Merck KGaA) in 6 -well plates at a density of $1-5 \times 10^{5} / \mathrm{ml}$, fixed in $4 \%$ paraformaldehyde for $15 \mathrm{~min}$, washed with PBS and permeabilized with $0.1 \%$ Triton-X-100 (Sigma-Aldrich) for $5 \mathrm{~min}$, all at room temperature. Subsequently, the cells were incubated with LC3-II primary antibody for $1 \mathrm{~h}$ at room temperature $(1: 1,000$; cat. no. L8918; Sigma-Aldrich; Merck KGaA), followed by incubation with a secondary antibody [fluorescein isothiocyanate (FITC)-conjugated; 1:100; cat. no. KC-RB-095; KangCheng Biotech, Shanghai, China] at room temperature for $1 \mathrm{~h}$. Cells were then incubated in the dark with DAPI (Invitrogen; Thermo Fisher Scientific, Inc.) for 5 min. After washing with PBS, glass slides were sealed with a seal sheet containing a fluorescence-quenching agent. A laser-scanning confocal fluorescence microscope (Olympus BX60; Olympus Corporation, Tokyo, Japan) was used to capture images.

Western blot analysis. Cells were lysed with cell lysis buffer (radioimmunoprecipitation assay buffer: phenylmethylsulfonyl
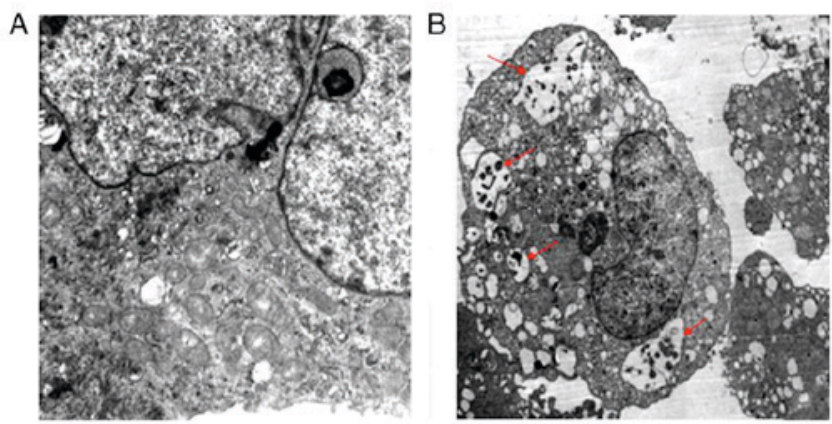

Figure 1. Enhanced autophagy in HK-2 cells following exposure to iohexol Transmission electron microscopy (magnification, x12,000) detected (A) normal cytoplasm and no autophagosomal formation in the control group. (B) Autophagic vesicles were observed in HK-2 cells following iohexol (200 mg iodine/ml) treatment for $6 \mathrm{~h}$. Arrows indicate typical autophagosomes, which contained the characteristic double membrane.

fluoride, 100:1; Beyotime Institutes of Biotechnology, Shanghai, China) on ice for $30 \mathrm{~min}$ and were centrifuged at $8,500 \mathrm{x} \mathrm{g}$ for $5 \mathrm{~min}$ at $4^{\circ} \mathrm{C}$. The protein concentration was determined using the Bicinchoninic Acid Protein Assay Reagent kit (Beyotime Institutes of Biotechnology). Proteins $(20 \mu \mathrm{g})$ were separated by $10 \%$ SDS-PAGE and were transferred to polyvinylidene fluoride membranes (EMD Millipore, Billerica, MA, USA). The membranes were blocked with calf serum (Gibco; Thermo Fisher Scientific, Inc.) for $1 \mathrm{~h}$ at room temperature and incubated with anti-LC3-II (1:1,000; cat. no. L8918; Sigma-Aldrich; Merck KGaA), anti-Atg7 (1:200; cat. no. A2856; Sigma-Aldrich; Merck KGaA) and anti- $\beta$-actin antibodies (1:2,000; cat. no. sc-7210; Santa Cruz Biotechnology, Inc., Dallas, TX, USA) overnight at $4^{\circ} \mathrm{C}$, followed by incubation with a secondary antibody $(1: 2,000$; cat. no. A0545; Sigma-Aldrich; Merck KGaA) for $1 \mathrm{~h}$ at room temperature. Enhanced chemiluminescent reagent (EMD Millipore) was used for chemiluminescence detection. The Kodak Gel Logic 200 Imaging System (Kodak, Rochester, NY, USA) was used to analyze the results.

Cell injury assay. HK-2 cells were cultured in 24-well plates at a density of $1-5 \times 10^{5} / \mathrm{ml}$. Once they reached $70-80 \%$ confluence, the cells were incubated with serum-free medium for $12 \mathrm{~h}$ and treated with iohexol and 3-MA as indicated. Cell injury was assessed using a lactate dehydrogenase (LDH) assay (Beyotime Institutes of Biotechnology) and an automatic biochemistry analyzer (Abbott Laboratories, Chicago, IL, USA).

MTT assay. An MTT assay (Sigma-Aldrich; Merck KGaA) was used to assess the viability and proliferation of HK-2 cells (4). Briefly, confluent HK-2 monolayers were incubated for $6 \mathrm{~h}$ with control medium, $200 \mathrm{mg} / \mathrm{ml}$ iohexol, or $200 \mathrm{mg} / \mathrm{ml}$ iohexol + $10 \mathrm{mM}$ 3-MA. Subsequently, $20 \mu \mathrm{l}(5 \mathrm{mg} / \mathrm{ml}) \mathrm{MTT}$ was added to the wells and was incubated for $4 \mathrm{~h}$ at $37^{\circ} \mathrm{C}$, after which $150 \mu \mathrm{l}$ dimethyl sulfoxide was added and oscillated for $10 \mathrm{~min}$. Cell viability was measured using a Labsystems Wellscan MK2 (Thermo Fisher Scientific, Inc.) at a 490-nm wavelength.

Cell apoptosis assay. Cell apoptosis was detected with an Annexin V FITC-conjugated/propidium iodide (PI) apoptosis kit (Nanjing Keygen Biotech Co., Ltd., Nanjing, China) 
A
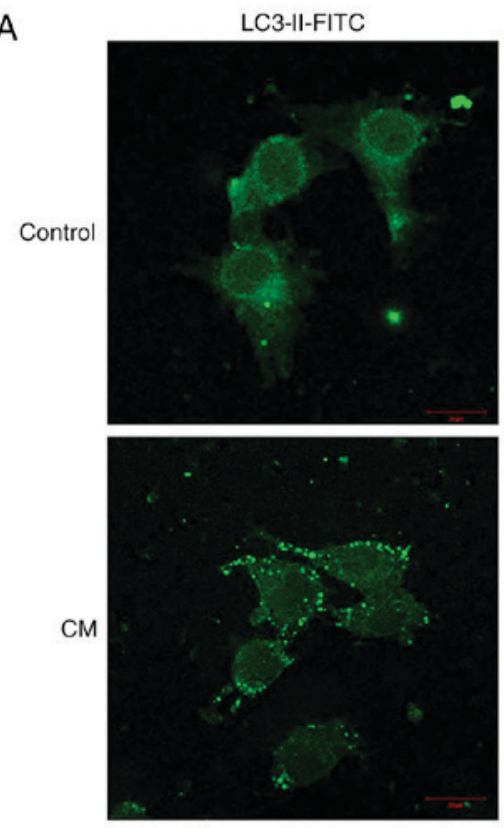

B

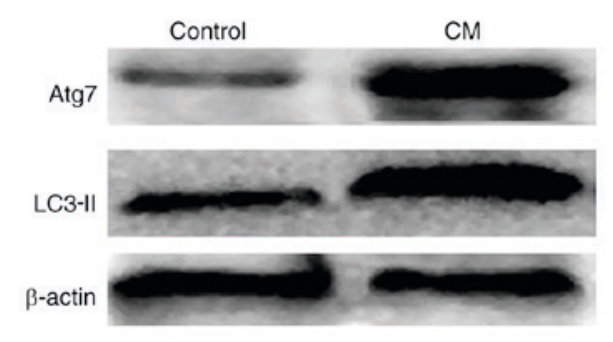

DAPI
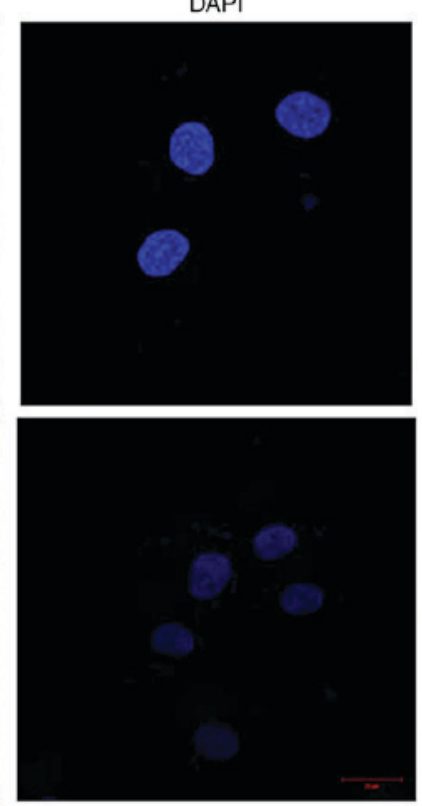
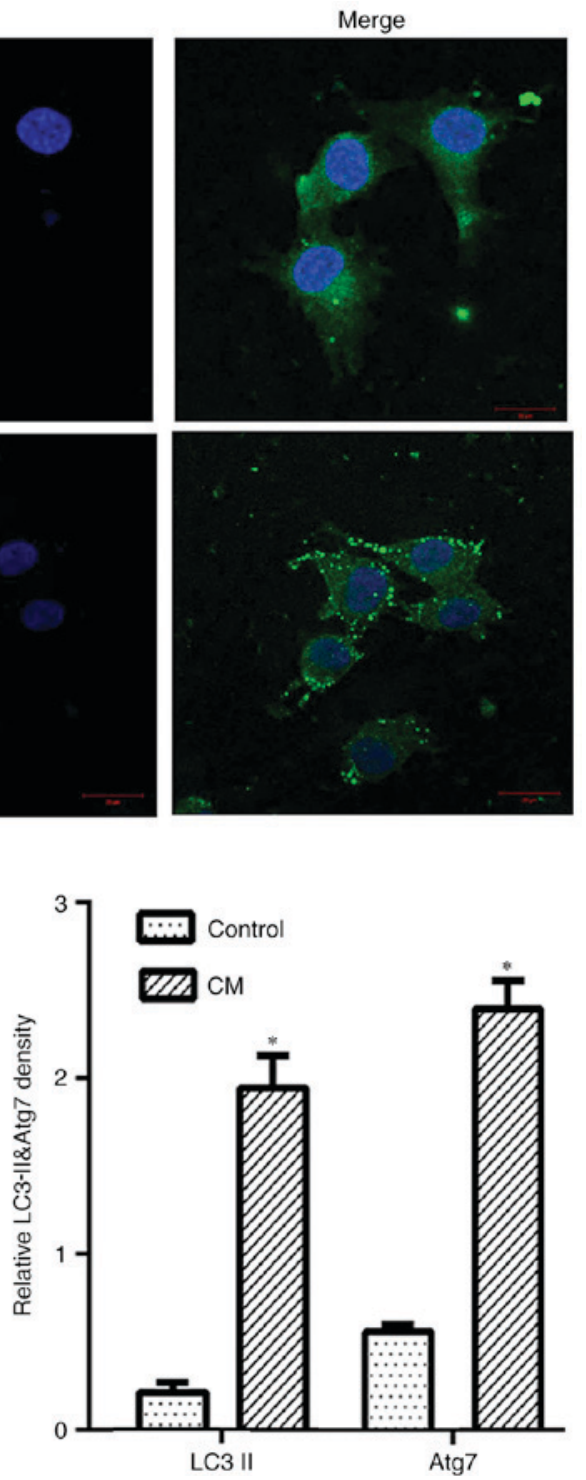

Figure 2. Autophagy was increased by iohexol. (A) Following treatment, cells were stained for LC3-II-FITC (green) and the nuclear marker DAPI (blue). Fluorescence micrographs (magnification, x630) demonstrated that treatment with iohexol markedly increased the levels of autophagy in HK-2 cells. (B) Western blot analysis was used to detect the protein expression levels of LC3-II and Atg7. "P<0.05 vs. the control group (n=6 experiments). Atg7, autophagy-related protein 7; CM, contrast media; FITC, fluorescein isothiocyanate; LC3-II, microtubule-associated protein 1A/1B-light chain 3-II.

A
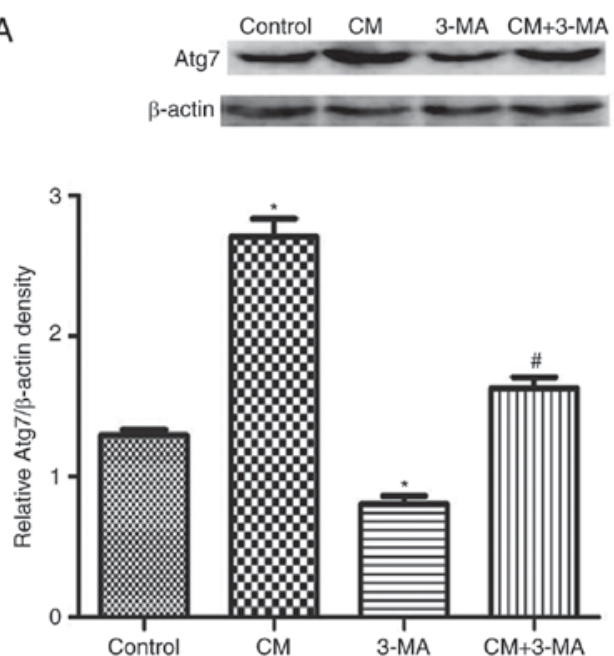

B
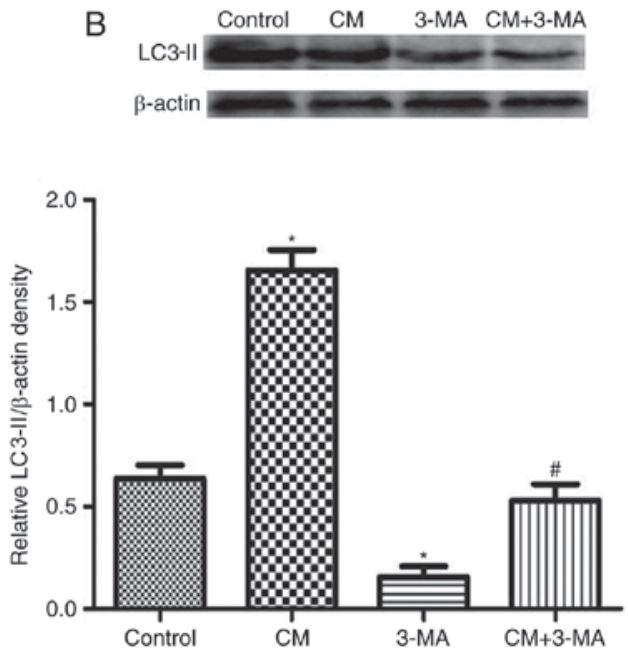

Figure 3. 3-MA inhibits autophagy in HK-2 cells treated with iohexol. Protein expression levels of (A) LC3-II and (B) Atg7 were detected by western blotting. ${ }^{\text {"P }}<0.05$ vs. the control group, ${ }^{\#} \mathrm{P}<0.01$ vs. the $\mathrm{CM}$ group ( $\mathrm{n}=6$ experiments). 3-MA, 3-methyladenine; Atg7, autophagy-related protein 7; CM, contrast media; LC3-II, microtubule-associated protein 1A/1B-light chain 3-II. 
using flow cytometry. Cells were seeded in a 6 -well plate at $2 \times 10^{5}$ cells/well. Once the cells reached $70-80 \%$ confluence, they were incubated with serum-free medium for $12 \mathrm{~h}$ and treated with iohexol and 3-MA as aforementioned. Subsequently, the cells were collected and incubated with $5 \mu$ 1 Annexin V-FITC and $5 \mu \mathrm{lPI}(50 \mathrm{mg} / \mathrm{ml})$ for $15 \mathrm{~min}$ in the dark at room temperature. The cells were immediately analyzed using a flow cytometer (BD Biosciences, San Jose, CA, USA).

Statistical analysis. SPSS software (version 17.0; SPSS, Inc., Chicago, IL, USA) was used to conduct statistical analysis. Data are presented as the mean \pm standard deviation from 6 independent experiments. For comparisons between any two groups, an unpaired Student's t-test was performed. Comparisons between data from more than two groups were assessed by one-way analysis of variance followed by Fisher's least significant difference test for post hoc comparisons. $\mathrm{P}<0.05$ was considered to indicate statistically significant difference.

\section{Results}

Iohexol promotes autophagy in $\mathrm{HK}-2$ cells. The present study used TEM to detect the effects of iohexol on autophagy. As presented in Fig. 1A, in the control group, the HK-2 cells exhibited normal cytoplasm with no autophagosomal formation. However, a marked accumulation of autophagosomes was observed in the iohexol-treated group; cytoplasmic material and/or membrane vesicles were encapsulated in vacuoles (red arrows; Fig. 1B).

Iohexol upregulates LC3-II and Atg7 expression levels. LC3 is a representative autophagosome marker, which is essential for the formation of autophagosomes. During autophagy, the cytosolic form of LC3 (LC3-I) is converted to LC3-II by Atg7 (E1-like enzyme) and Atg3 (E2-like enzyme) (8). In the present study, the results of an immunofluorescence assay demonstrated that LC3-II expression was evidently upregulated in the cytoplasm by iohexol (Fig. 2A). Furthermore, these observations were confirmed by western blot analysis. As shown in Fig. 2B, LC3-II and Atg7 expression were markedly upregulated by iohexol (200 $\mathrm{mg}$ iodine/ml) compared with the control group $(\mathrm{P}<0.05)$. These results indicated that iohexol may increase the levels of autophagy in HK-2 cells.

3-MA suppresses iohexol-induced autophagy. 3-MA, which is an inhibitor of phosphatidylinositol 3-kinase, was used to inhibit the initiation of autophagosome formation. As presented in Fig. 3, 3-MA not only affected the expression levels of LC3-II and Atg7 in HK-2 cells compared with the control group, but also reversed iohexol-induced upregulation of LC3-II and Atg7 expression (Fig. 3).

Autophagy serves a protective role in iohexol-induced cytotoxicity. As presented in Fig. 4 and Table I, the cytotoxicity of iohexol was evaluated by MTT assay and LDH release. LDH levels were significantly increased $(\mathrm{P}<0.05)$ and cell viability was significantly decreased in the iohexol-treated group compared with the control group, which was further enhanced by 3-MA.
Table I. Effects of 3-MA on cell proliferation and viability in iohexol-treated HK-2 cells (mean \pm standard deviation, $n=6$ ).

\begin{tabular}{lc}
\hline Group & MTT assay result (OD value) \\
\hline Control & $0.585 \pm 0.003$ \\
CM & $0.345 \pm 0.001^{\mathrm{a}}$ \\
3 -MA & $0.534 \pm 0.002$ \\
CM + 3-MA & $0.216 \pm 0.004^{\mathrm{b}}$ \\
\hline
\end{tabular}

${ }^{\mathrm{a}} \mathrm{P}<0.01$ vs. the control group; ${ }^{\mathrm{b}} \mathrm{P}<0.05$ vs. the $\mathrm{CM}$ group. $3-\mathrm{MA}$, 3-methyladenine; $\mathrm{CM}$, contrast media; OD, optical density.

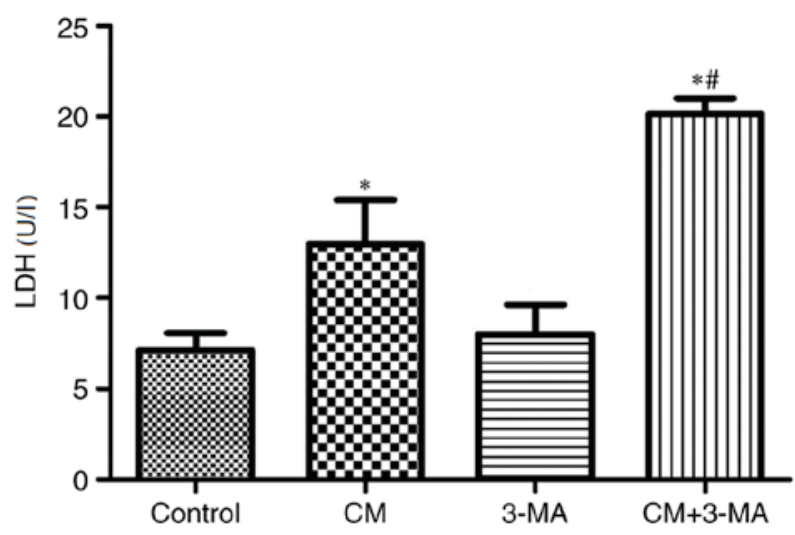

Figure 4. Protective effects of autophagy on the cytotoxicity of HK-2 cells induced by iohexol. Iohexol-induced LDH release was enhanced by 3-MA. ${ }^{\text {"}} \mathrm{P}<0.05$ vs. the control group, ${ }^{\prime} \mathrm{P}<0.01$ vs. the $\mathrm{CM}$ group ( $\mathrm{n}=6$ experiments). 3-MA, 3-methyladenine; CM, contrast media; LDH, lactate dehydrogenase.

Autophagy serves a protective role in iohexol-induced apoptosis. The role of autophagy in iohexol-induced HK-2 cell apoptosis was analyzed (Figs. 5 and 6). As shown in Figs. 5B and 6, iohexol significantly increased apoptosis of HK-2 cells compared with the control group. Treatment with 3-MA alone did not affect cell apoptosis (Figs. 5C and 6). However, cell apoptosis was significantly increased in the iohexol + 3-MA group compared with the iohexol group (Figs. 5D and 6).

\section{Discussion}

CIN is a serious complication resulting from the use of iodinated $\mathrm{CM}$; however, the mechanism underlying CIN remains to be elucidated. A previous study has indicated that the pathogenesis of CIN is predominantly associated with renal ischemia, direct nephrotoxicity and oxidative stress (4). Autophagy is a cellular stress response that serves important roles in the pathogenesis of various diseases. Numerous studies have demonstrated that autophagy is closely associated with apoptosis $(7,13,14)$. In some cases, cell apoptosis induced by cellular stress may be reduced by autophagy; however, excessive autophagy can also contribute to cell death $(13,15)$. A previous study reported that $\mathrm{CM}$ induced organelle damage, which may result in the activation of autophagy (10). When CM was injected intravenously, it was filtered by glomerular filtration and directly damaged renal tubular epithelial cells. An in vitro study suggested that the number of lysosomes was increased 

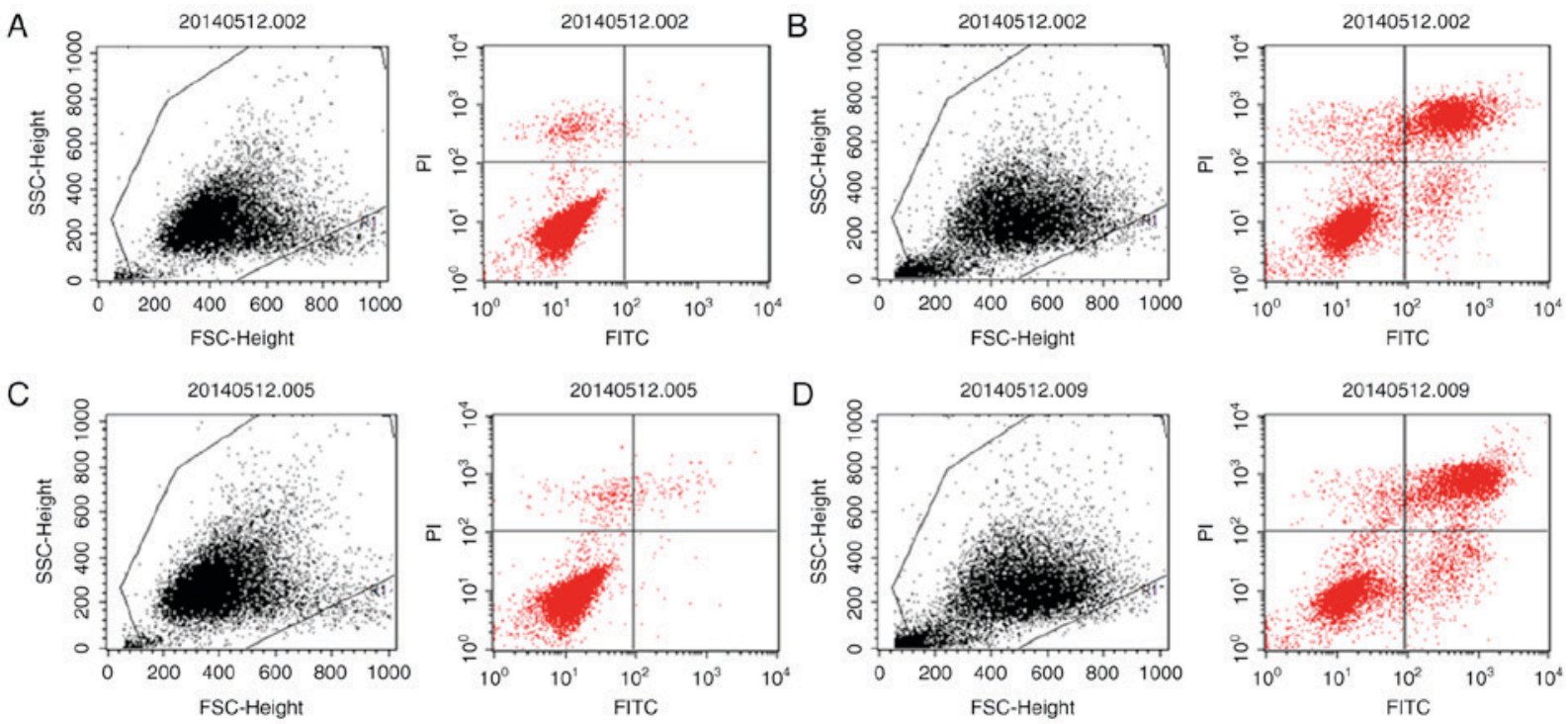

Figure 5. Representative results of the percentage of apoptotic HK-2 cells, as determined using flow cytometry. (A) Control; (B) CM; (C) 3-MA; and (D) CM + 3-MA groups. 3-MA, 3-methyladenine; CM, contrast media.

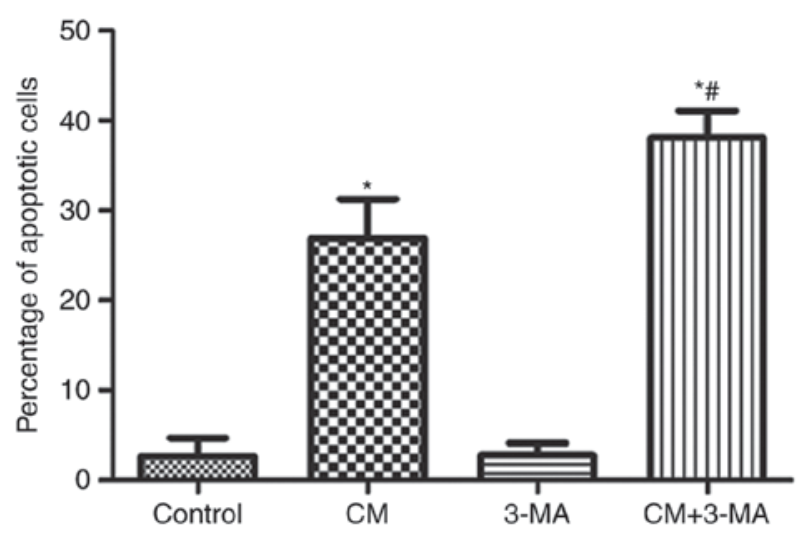

Figure 6. Effects of 3-MA on the percentage of apoptotic HK-2 cells induced by iohexol and 3-MA. Iohexol-induced apoptosis was enhanced by 3-MA. ${ }^{*} \mathrm{P}<0.05$ vs. the control group, ${ }^{\#} \mathrm{P}<0.01$ vs. the $\mathrm{CM}$ group ( $\mathrm{n}=6$ experiments). 3-MA, 3-methyladenine; CM, contrast media.

in the cells of the proximal convoluted tubule following CM injection (16). In addition, CM has been reported to reduce cell proliferation, alter mitochondrial function and increase apoptosis of proximal tubule cells (17). Buyuklu et al (18) suggested that oxidative stress, inflammation, autophagy and apoptosis are increased in rats with CIN. Consistent with these results, our previous study demonstrated that CM increased cell apoptosis and reduced cell viability in a time- and dose-dependent manner (5). The present study detected a marked accumulation of autophagosomes in iohexol-treated cells. LC3-II and Atg7 expression were markedly upregulated by iohexol $(200 \mathrm{mg}$ iodine $/ \mathrm{ml}$ ) compared with the control cells. These results indicated that iohexol markedly increased the levels of autophagy in HK-2 cells, which further supported the hypothesis that CM induces activation of cell autophagy.

Autophagy is a fundamental cellular homeostatic process that is used to degrade and recycle cellular proteins, and remove damaged organelles. Previous studies have demonstrated that autophagy serves a protective role in AKI $(10,19)$.
Inhibition of autophagy by autophagy inhibitors has been reported to evidently downregulate cisplatin-induced apoptosis in proximal tubular cells (20). Jiang et al (10) reported that proximal tubule-specific autophagy-deficient mice developed more severe AKI and increased cisplatin-induced apoptosis. In another experimental model of cisplatin-induced nephropathy, autophagy was reported to have a role in cell death, and a precise balance between apoptosis and autophagy was detected (21). Autophagy contributes to renoprotection via the modulation of apoptosis and mitochondrial injury in an animal model of CIN; in vitro studies also demonstrated that decreased cell viability by iohexol (100 $\mathrm{mg}$ iodine/ml) was aggravated with 3-MA pretreatment (19). However, the effects of autophagy on iohexol-induced apoptosis of HK-2 cells remain unclear. The present study demonstrated that the protein expression levels of LC3-II and Atg7 were upregulated in HK-2 cells by iohexol. When HK-2 cells were treated with iohexol for $6 \mathrm{~h}$, autophagosomes were accumulated in the cytoplasm, thus suggesting that iohexol induced autophagy in HK-2 cells. Furthermore, inhibition of autophagy by 3-MA evidently enhanced iohexol-induced cell injury and apoptosis of HK-2 cells. These results indicated that activation of autophagy may attenuate iohexol-induced cytotoxicity, thus suggesting that iohexol-induced activation of autophagy may serve a protective role in iohexol-induced cytotoxicity of renal tubular epithelial cells. The potential mechanisms were hypothesized as follows: Autophagy may provide adenosine triphosphate for energy-deficient cells by consuming aged and damaged organelles or proteins; autophagy may serve a critical role in removing protein aggregates and damaged organelles, and may promote cell survival and tissue homeostasis; autophagy may reduce apoptosis of cells by intervening with apoptotic signaling pathways.

In conclusion, the present study revealed that autophagy was upregulated by iohexol in HK-2 cells, and confirmed that iohexol-induced autophagy may attenuate cell injury and the apoptosis of HK-2 cells. These findings suggested that autophagy serves a protective role in the CM-induced injury 
of renal tubular epithelial cells. Further studies are required to explore the specific mechanisms underlying the effects of autophagy on iohexol-induced cytotoxicity in HK-2 cells.

\section{Acknowledgements}

The present study was supported by the National Natural Science Foundation of China (grant no. 81570618) and the Scientific Foundation of Hunan Province, China (grant nos. 2010FJ6008 and 2008JT3005). The authors would like to thank Dr Yiya Zhang (The Key Laboratory of Protein Chemistry and Developmental Biology of Ministry of Education, College of Life Sciences, Hunan Normal University, Changsha, China) for reviewing the manuscript.

\section{References}

1. Sun S, Zhang T, Nie P, Hu L, Yu Y, Cui M, Cai Z, Shen L and He B: A novel rat model of contrast-induced acute kidney injury. Int J Cardiol 172: e48-e50, 2014.

2. Solomon RJ, Mehran R, Natarajan MK, Doucet S, Katholi RE, Staniloae CS, Sharma SK, Labinaz M, Gelormini JL and Barrett BJ: Contrast-induced nephropathy and long-term adverse events: Cause and effect? Clin J Am Soc Nephrol 4: 1162-1169, 2009.

3. Nazıroğlu M, Yoldaş N, Uzgur EN and Kayan M: Role of contrast media on oxidative stress, $\mathrm{Ca}(2+)$ signaling and apoptosis in kidney. J Membr Biol 246: 91-100, 2013.

4. Quintavalle C, Brenca M, De Micco F, Fiore D, Romano S, Romano MF, Apone F, Bianco A, Zabatta MA, Troncone G, et al: In vivo and in vitro assessment of pathways involved in contrast media-induced renal cells apoptosis. Cell Death Dis 2: e155, 2011.

5. Duan S, Zhou X, Liu F, Peng Y, Chen Y, Pei Y, Ling G, Zhou L, Li Y, Pi Y, et al: Comparative cytotoxicity of high-osmolar and low-osmolar contrast media on HKCs in vitro. J Nephrol 19: 717-724, 2006.

6. Lamb CA, Yoshimori $\mathrm{T}$ and Tooze SA: The autophagosome: Origins unknown, biogenesis complex. Nat Rev Mol Cell Biol 14: 759-774, 2013.

7. Yang C, Kaushal V, Shah SV and Kaushal GP: Autophagy is associated with apoptosis in cisplatin injury to renal tubular epithelial cells. Am J Physiol Renal Physiol 294: F777-F787, 2008.
8. Howell GM, Gomez H, Collage RD, Loughran P, Zhang X, Escobar DA, Billiar TR, Zuckerbraun BS and Rosengart MR: Augmenting autophagy to treat acute kidney injury during endotoxemia in mice. PLoS One 8: e69520, 2013.

9. Wang $\mathrm{Z}$ and Choi ME: Autophagy in kidney health and disease. Antioxid Redox Signal 20: 519-537, 2014.

10. Jiang M, Wei Q, Dong G, Komatsu M, Su Y and Dong Z: Autophagy in proximal tubules protects against acute kidney injury. Kidney Int 82: 1271-1283, 2012.

11. Liu GL, Lei R, Duan SB, Tang MM, Luo M and Xu Q: Atorvastatin alleviates iodinated contrast media-induced cytotoxicity in human proximal renal tubular epithelial cells. Exp Ther Med: August 1, 2017 (Epub ahead of print). doi: 10.3892/etm.2017.4859.

12. Winey M, Meehl JB, O'Toole ET and Giddings TH Jr: Conventional transmission electron microscopy. Mol Biol Cell 25: 319-323, 2014.

13. Maiuri MC, Zalckvar E, Kimchi A and Kroemer G: Self-eating and self-killing: Crosstalk between autophagy and apoptosis. Nat Rev Mol Cell Biol 8: 741-752, 2007.

14. Ding Y and Choi ME: Autophagy in diabetic nephropathy. J Endocrinol 224: R15-R30, 2015.

15. Rubinstein AD and Kimchi A: Life in the balance-a mechanistic view of the crosstalk between autophagy and apoptosis. J Cell Sci 125: 5259-5268, 2012.

16. Tervahartiala P, Kivisaari L, Kivisaari R, Vehmas $T$ and Virtanen I: Structural changes in the renal proximal tubular cells induced by iodinated contrast media. Nephron 76: 96-102, 1997.

17. Hardiek K, Katholi RE, Ramkumar V and Deitrick C: Proximal tubule cell response to radiographic contrast media. Am J Physiol Renal Physiol 280: F61-F70, 2001.

18. Buyuklu M, Kandemir FM, Ozkaraca M, Set T, Bakirci EM, Topal E, lleriturk M and Turkmen K: Benefical effects of lycopene against contrast medium-induced oxidative stress, inflammation, autophagy, and apoptosis in rat kidney. Hum Exp Toxicol 34: 487-496, 2015.

19. Ko GJ, Bae SY, Hong YA, Pyo HJ and Kwon YJ: Radiocontrast-induced nephropathy is attenuated by autophagy through regulation of apoptosis and inflammation. Hum Exp Toxicol 35: 724-736, 2016.

20. Periyasamy-Thandavan S, Jiang M, Wei Q, Smith R, Yin XM and Dong Z: Autophagy is cytoprotective during cisplatin injury of renal proximal tubular cells. Kidney Int 74: 631-640, 2008.

21. Domitrović R, Cvijanović O, Pernjak-Pugel E, Skoda M, Mikelić L and Crnčević-Orlić Z: Berberine exerts nephroprotective effect against cisplatin-induced kidney damage through inhibition of oxidative/nitrosative stress, inflammation, autophagy and apoptosis. Food Chem Toxicol 62: 397-406, 2013. 